\title{
Effects of Verbal Information in Background Music on Mental Task and its Relation to Cerebral Blood Flow
}

\author{
Naruki Shirahama ${ }^{\mathrm{a},{ }^{*}}$, Takahiro Higashi ${ }^{\mathrm{a}}$, and Satoshi Watanabe ${ }^{\mathrm{b}}$ \\ ${ }^{\text {a }}$ Department of Creative Engineering, National Institute of Technology, Kitakyushu College \\ 5-20-1, Shii, Kokuraminami-ku, Kitakyushu-shi, Fukuoka 802-0985, Japan \\ ${ }^{\mathrm{b}} \mathrm{School}$ of Health Sciences at Odawara, International University of Health and Welfare \\ 1-2-25, Shiroyama, Odawara-shi, Kanagawa 250-8588, Japan \\ *Corresponding Author: naruki@kct.ac.jp
}

\begin{abstract}
In today's society, there is a lot of desk work, and in schools and workplaces, people need to be able to perform various tasks efficiently and with concentration. The work environment has a significant impact on the efficiency of desk work. This study investigates the effects of background music on work and cerebral blood flow during mental tasks. In this study, we used NIRS to measure and verify changes in cerebral blood flow in collaborators who calculated and memorized English words while listening to background music. In particular, we hypothesized and tested that the presence or absence of vocals in the background music would affect cerebral blood flow. We adopted a block design as our experimental method for measuring cerebral blood flow. The descriptive statistics values, maximum, minimum, mean, and variance, were calculated from the measurement results of cerebral blood flow, and a box plot represented the size distribution. The experimental results showed that the variance of cerebral blood flow between collaborators was more significant during computational tasks than at rest. The group that contained verbal information had higher overall cerebral blood flow than the group that did not. Besides, cerebral blood flow was lower during the task than during rest.
\end{abstract}

Keywords: background music, NIRS, calculation task, memory task, block design

\section{Introduction}

When we perform any work, we need to do it quickly and with as few mistakes as possible. Various environmental factors influence work efficiency and research on the relationship between work efficiency and environmental factors has been conducted extensively for a long time ${ }^{(1)}$. Arai et al. experimented with comparing the effects of background music on the efficiency of simple tasks between classical and pop music and found that classical music activated cerebral blood flow more than pop music, as measured by NIRS ${ }^{(2)}$. Suga et al. examined the effects of three conditions: uplifting music, depressive music, and no music as a control condition, on the performance of a 4-digit/2-digit calculation task by university students ${ }^{(3)}$. The results showed that the three conditions had neither facilitating nor interfering effects on workload, fatigue, and motivation. On the other hand, there was a statistically different tendency among the three conditions in the scale of an impression of work.

In addition, research has been conducted focusing on what is called "studying while watching TV or listening to music" in everyday learning situations. Suga et al. also experimented with comparing the presence or absence of music presentation during work for a group of students who had the habit of studying while listening to music and students who did not ${ }^{(4)}$. There was no significant difference in the number of answers, correct answers, or wrong answers due to habit differences. However, it was found that music can be a desirable learning environment for students who have the habit of studying while listening to music, but an undesirable learning environment for students 
who do not have this habit. Abe et al. conducted an experiment focusing on the difference in tempo of background music ${ }^{(5)}$. The experiment showed that for three types of tasks with different cognitive loads, the tempo did not affect the high and medium load tasks, while the tempo increased the work efficiency for the low load tasks. Ferreri et al. used a 48-channel NIRS system to monitor the dorsolateral prefrontal cortex and suggested that background music positively affects both episodic encoding and retrieval of verbal information ${ }^{(6)}$.

The purpose of our research is to create an efficient learning environment. In particular, we focused on background music to examine the necessity of background music and, if necessary, what kind of background music is appropriate. In particular, the following two points were set as research questions in this study.

1. To measure changes in cerebral blood flow at rest and during mental tasks while listening to background music.

2. To investigate the effect of verbal information (e.g., vocals of songs) in background music on mental tasks.

\section{Near-Infrared Spectroscopy Measurement}

Near-infrared spectroscopy (NIRS) is a non-invasive method of measuring changes in blood flow in the brain using near-infrared light. In this study, we used NeU's HOT-2000 as the NIRS system ${ }^{(7)}$.

The HOT-2000 measures changes in brain activity by placing light sources and photosensors at $3 \mathrm{~cm}$ intervals on the head. For the light source, we used near-infrared light in the wavelength band of $800 \mathrm{~nm}$. Near-infrared light penetrates human tissue but is absorbed by hemoglobin. Oxygen and glucose are necessary for brain nerve activity. Therefore, hemoglobin increases in the area where brain activity is active, and the transmission of NIR light decreases. Figure 1 shows an image of optical measurement of cerebral blood flow changes. Moreover, NIRS can visualize brain activity by measuring the change in light.

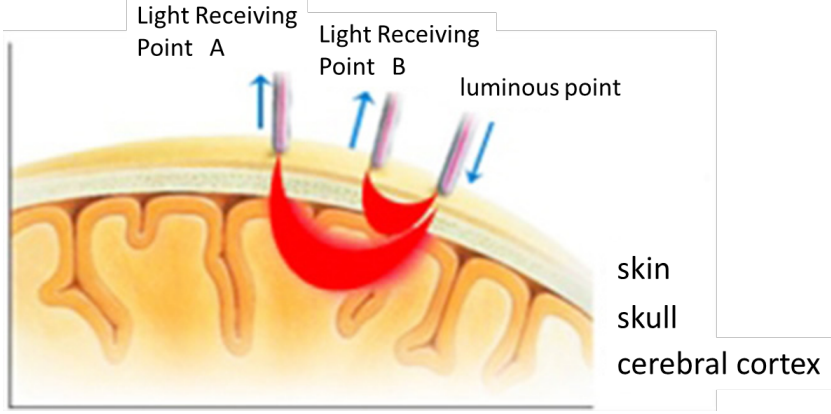

Fig. 1. An image of optical measurement of cerebral blood flow changes

(https://neu-brains.co.jp/service/equipments/hot-2000/)

\section{Experimental Methods}

\subsection{Overview}

The experiment was conducted on ten collaborators. First, we explained the experiment to the collaborators and obtained their written consent to participate in the experiment. In this experiment, we used NeU's HOT-2000 (2 measurement channels) as the NIRS instrument. The music used in this experiment was obtained with permission from "Maoudamashii," a website dealing with free sound source materials ${ }^{(8)}$.

Then, two types of mental tasks were performed on the collaborators: a calculation task to divide a four-digit number by a two-digit number and a memorization task of English words.

\subsection{Design of the Experiment}

In this experiment, we used a block design as the experimental method. The block design is an experimental method in which the same task and rest are repeated multiple times. The task in this experiment is a calculation task and an English word memorization task.

In this experiment, the task and rest periods were 30 seconds each for calculation and memory tasks. Five sets were performed within each task. The design of the blocks is shown in Figure 2. Each block was assigned a number from 1 to 10, as shown in Figure 2. 


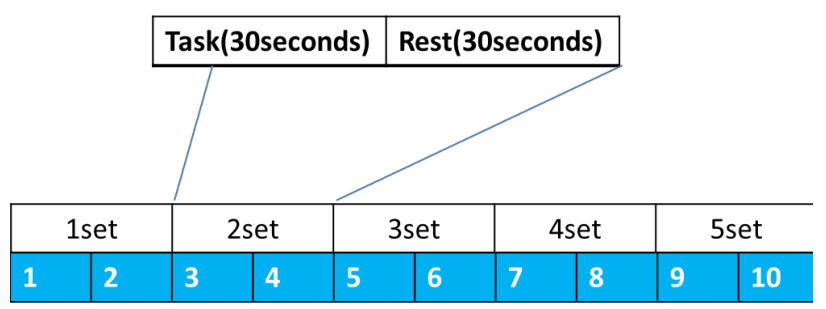

Fig. 2. The block design used in this experiment.

\subsection{Experimental Protocol}

The experimental protocol is as follows:

1. Collaborators are randomly divided into two groups: one with vocals and the other without vocals. The former group will be referred to as Group A and the latter as Group B.

2. Measure the cerebral blood flow of the collaborators at rest.

3. Collaborators are asked to perform a calculation task. During this time, measure the cerebral blood flow on both sides. After the measurement, the collaborators will be given a 5-minute break.

4. After the break, the collaborators will be asked to memorize English words. During this time, the cerebral blood flow is measured in both right and left hemispheres.

5. After the work, a simple English word test will be given to check the memorization status.

6. After the test, the participants will be given a questionnaire about the background music and each task.

\section{Experimental Results}

The maximum, minimum, mean, and variance values for each block in each collaborator were calculated based on the measured values of cerebral blood flow. These are shown in Tables 1 to 4 . The basic statistics of the resting state are shown in Table 5.

The distribution of cerebral blood flow of all collaborators in each block is shown in a box plot. The distribution of measured values in the left and right frontal channels are shown in Figure 3 and Figure 4, respectively. The color red indicates when the user listens to a song that includes vocals, and the color green indicates when the user listens to a song that does not include vocals.
Table 1. Maximum values hemoglobin concentration

\begin{tabular}{|c|c|c|c|c|}
\hline \multirow{3}{*}{$\begin{array}{l}\text { Block } \\
\text { number }\end{array}$} & \multicolumn{4}{|c|}{ Hemoglobin concentration[mMmm] } \\
\hline & \multicolumn{2}{|c|}{ Word memorization task } & \multicolumn{2}{|c|}{ Calculation task } \\
\hline & On vocal & Off vocal & On vocal & Off vocal \\
\hline 1 & 2.92 & 1.98 & 2.68 & 2.13 \\
\hline 2 & 1.97 & 1.78 & 2.02 & 1.66 \\
\hline 3 & 3.19 & 2.03 & 3.14 & 2.64 \\
\hline 4 & 2.18 & 1.64 & 1.87 & 1.90 \\
\hline 5 & 2.58 & 2.07 & 3.28 & 2.41 \\
\hline 6 & 1.58 & 1.75 & 1.89 & 1.67 \\
\hline 7 & 2.78 & 2.82 & 3.21 & 2.60 \\
\hline 8 & 1.47 & 2.11 & 1.49 & 1.60 \\
\hline 9 & 3.00 & 1.67 & 2.78 & 2.42 \\
\hline 10 & 2.23 & 1.63 & 1.28 & 1.59 \\
\hline
\end{tabular}

Table 2. Minimum values hemoglobin concentration

\begin{tabular}{|c|c|c|c|c|}
\hline \multirow{3}{*}{$\begin{array}{l}\text { Block } \\
\text { number }\end{array}$} & \multicolumn{4}{|c|}{ Hemoglobin concentration[mMmm] } \\
\hline & \multicolumn{2}{|c|}{ Word memorization task } & \multicolumn{2}{|c|}{ Calculation task } \\
\hline & On vocal & Off vocal & On vocal & Off vocal \\
\hline 1 & -1.57 & -0.39 & -0.16 & -0.26 \\
\hline 2 & -1.88 & -1.12 & -0.40 & -0.40 \\
\hline 3 & -1.58 & -1.06 & -1.24 & -0.37 \\
\hline 4 & -1.94 & -1.43 & -0.61 & -0.61 \\
\hline 5 & -1.76 & -1.29 & -0.97 & -0.49 \\
\hline 6 & -1.77 & -3.49 & -0.70 & -0.70 \\
\hline 7 & -1.53 & -1.40 & -1.22 & -0.58 \\
\hline 8 & -1.99 & -3.41 & -0.74 & -0.74 \\
\hline 9 & -1.84 & -1.41 & -0.64 & -0.64 \\
\hline 10 & -1.80 & -3.41 & -0.75 & -0.75 \\
\hline
\end{tabular}

Table 3. Mean values of hemoglobin concentration

\begin{tabular}{|c|c|c|c|c|c|}
\hline & \multicolumn{3}{|c}{ Hemoglobin concentration [mMmm] } \\
\cline { 2 - 3 } Block & Word memorization task & \multicolumn{2}{c|}{ Calculation task } \\
\cline { 2 - 3 } \cline { 5 - 5 } number & On vocal & Off vocal & & On vocal & Off vocal \\
\hline 1 & 0.82 & 0.75 & 0.77 & 0.42 \\
\hline 2 & 0.50 & 0.20 & 0.39 & 0.21 \\
\hline 3 & 1.03 & 0.35 & 0.94 & 0.52 \\
\hline 4 & 0.43 & 0.11 & 0.45 & 0.15 \\
\hline 5 & 0.91 & 0.36 & 1.07 & 0.42 \\
\hline 6 & 0.52 & 0.40 & 0.30 & 0.10 \\
\hline 7 & 1.03 & 0.45 & 0.82 & 0.37 \\
\hline 8 & 0.45 & -0.54 & 0.35 & 0.06 \\
\hline 9 & 1.02 & 0.27 & 0.86 & 0.39 \\
\hline 10 & 0.48 & -0.67 & 0.41 & 0.15 \\
\hline
\end{tabular}

Table 4. Variances of hemoglobin concentration

\begin{tabular}{|c|c|c|c|c|}
\hline & \multicolumn{3}{|c|}{ Hemoglobin concentration [mMmm] } \\
\cline { 2 - 3 } Block & Word memorization task & \multicolumn{2}{|c|}{ Calculation task } \\
\cline { 2 - 3 } \cline { 5 - 5 } number & On vocal & Off vocal & On vocal & Off vocal \\
\hline 1 & 2.66 & 0.57 & 2.92 & 0.71 \\
\hline 2 & 1.70 & 0.72 & 1.96 & 0.59 \\
\hline 3 & 2.41 & 0.97 & 2.79 & 1.19 \\
\hline 4 & 1.69 & 1.09 & 1.85 & 0.77 \\
\hline 5 & 1.90 & 1.28 & 2.96 & 1.11 \\
\hline 6 & 1.59 & 1.59 & 1.54 & 0.76 \\
\hline 7 & 2.07 & 1.86 & 2.84 & 1.31 \\
\hline 8 & 1.68 & 3.41 & 1.34 & 0.74 \\
\hline 9 & 2.73 & 1.21 & 2.11 & 1.22 \\
\hline 10 & 1.74 & 2.80 & 1.25 & 0.75 \\
\hline
\end{tabular}


Table 5. Hemoglobin concentration at rest

\begin{tabular}{c|c|c}
\hline & On vocal & Off Vocal \\
\hline \hline Max & 1.81 & 1.68 \\
\hline Min & -1.67 & -0.221 \\
\hline Mean & 0.489 & 0.271 \\
\hline Variances & 1.75 & 0.467 \\
\hline
\end{tabular}

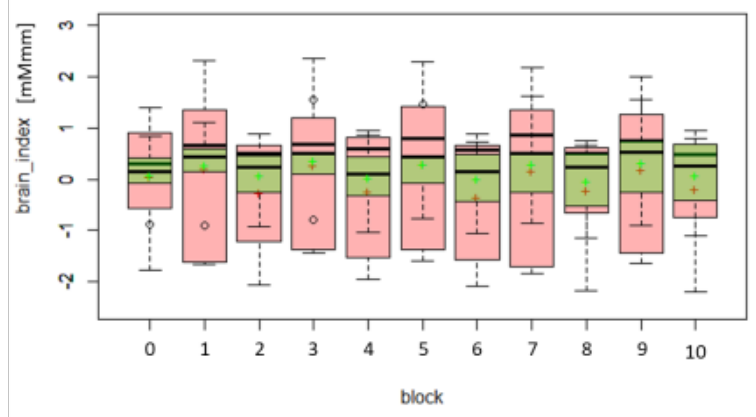

Fig.3 Calculation work (left in front)

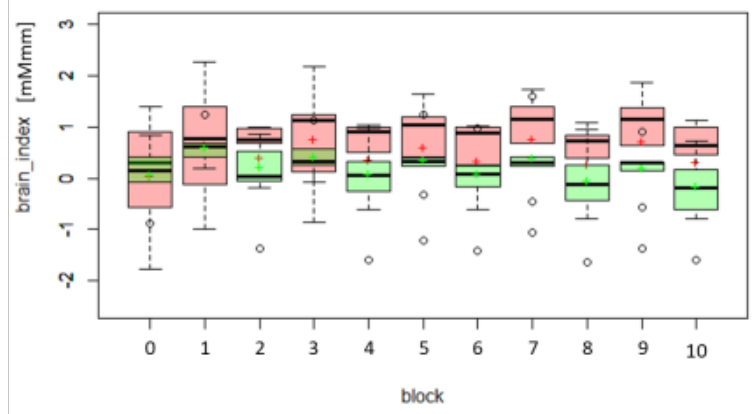

Fig.4 English words memorization work (left in front)

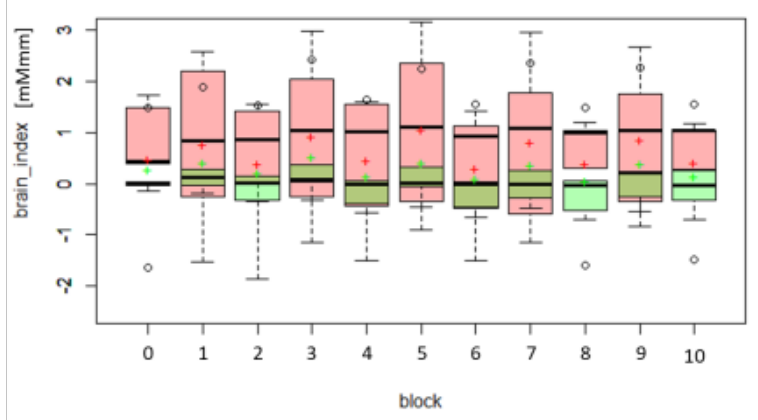

Fig.5 Calculation work (right in front)

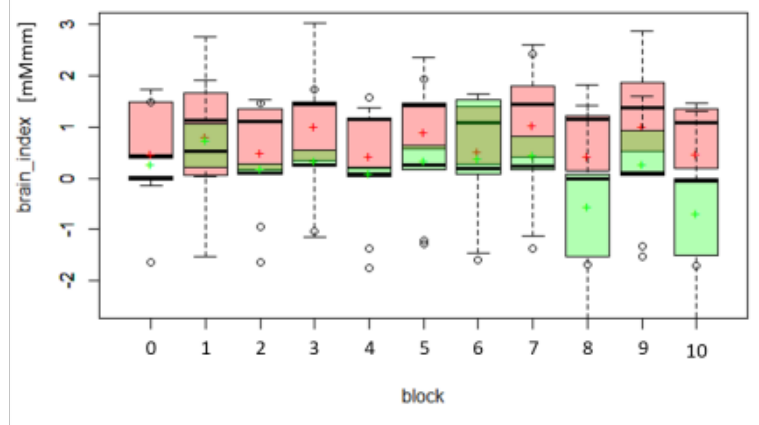

Fig.6 English words memorization work (right in front)

The average value for each block is plotted as "+." (As a supplement, 0 on the horizontal axis represents the measured resting cerebral blood flow measured in experimental protocol 2.)

In both Figure 3 and Figure 4, Group A has higher blood flow than Group B. These figures show that the variation in cerebral blood flow among the subjects is considerable during computational tasks. Regardless of the group or task, blood flow tends to increase during task execution compared to rest.

\section{Conclusions}

The purpose of this experiment was to confirm the changes in cerebral blood flow during mental tasks at rest and while listening to background music and then to investigate the influence of linguistic information on background music on mental tasks. The results of this experiment confirmed that "individual differences in cerebral blood flow are greater during task execution than during resting" and that "cerebral blood flow increases more when working while listening to music with vocals than when listening to music without vocals."

However, the number of collaborators in this preliminary experiment was relatively small, and the number of collaborators is insufficient to draw any conclusions. In addition, we found that cerebral blood flow increased during task execution compared to rest, but the difference seemed to be small. In the present study, we could only visualize the obtained data by box plot, so we could not clarify whether the difference was significant or not. This experiment was conducted with a small number of collaborators, and it can be said that it was to confirm the performance of the measurement equipment. In the future, we will increase the number of collaborators and analyze 
the data using statistical methods such as hypothesis testing and clustering.

\section{References}

(1) Mamoru Umemura, and Naoyuki Aizawa : "Influence of noise on mental work", The Japanese Journal of Ergonomics, Vol. 22, No. 5, pp. 259-268, 2010. (in Japanese), https://doi.org/10.5100/jje.22.259

(2) Arai Yoshihiko, and Kashikura Kenichi : "Brain Activation Related to Work Efficiency While Listening to Background Music", Departmental Bulletin Paper, Gunma Prefectural College of Health Sciences, Vol. 7, pp. 45-53, 2012. (in Japanese) http://id.nii.ac.jp/1725/00000061/

(3) Sensaku Suga, and Yousuke Iwamoto : "Effects of Background Music on Calculation Task - From Cognitive and Affective Viewpoints -", Bulletin of the Faculty of Education, Wakayama University. Educational science, Vol.13, pp. 27-36, 2003. (in Japanese) http://repository.center.wakayama-u.ac.jp/378

(4) Sensaku Suga, Junko Goto : "Effects of Background Music on Calculation and Memory Tasks - Diffrences in the Subjects' Habit of Daily Study: 'With' or 'Without' Music -", Bulletin of the Faculty of Education, Wakayama University. Educational science, Vol.18, pp. 59-68, 2008. (in Japanese) http://repository.center.wakayama-u.ac.jp/487

(5) Asami Abe, and Noriko Shingaki : "The Effect of the Tempo of background music on Work Efficiency", The Proceedings of the 27th annual meeting of Japanese Cognitive Science Society, pp. 853-859, 2010. (in Japanese)

(6) Ferreri L, Bigand E, Bard P, Bugaiska : “A. The Influence of Music on Prefrontal Cortex during Episodic Encoding and Retrieval of Verbal Information: A Multichannel fNIRS Study", Behavioural Neurology, Volume 2015, Article ID 707625, 11 pages, 2015. http://dx.doi.org/10.1155/2015/707625

(7) Neuroscience Company Corporation : Brain Imaging Hardware (NIRS) \& Systems (in Japanese) https://neu-brains.co.jp

(8) Koichi Morita : Maoudamashii (in Japanese) https://maoudamashii.jokersounds.com/ 